\title{
Study of Kinesthetic Negotiation Ability in Lightweight Comanipulative Decision-making Tasks: Design and Study of a Virtual Partner based on Human-human Interaction Observation
}

LUCAS ROCHE and LUDOVIC SAINT-BAUZEL, Institut des Systèmes Intelligents et de Robotique (ISIR) - UMR7222 Sorbonne Université, CNRS, INSERM (U1150), France

This article presents the results of an experiment on physical Human-Human Interaction (pHHI), where human dyads cooperate on a one-dimensional comanipulative task in a novel lightweight teleoperation setup. The results of this experiment show that humans are able to handle asymmetrical information about the task and solve conflicts using only the kinesthetic channel. Data from the pHHI experiment is used to design a virtual partner that can perform the task alongside a human. The virtual partner behavior is based on the observation that initiative is highly correlated to decision-making in our pHHI negotiation scenario. The virtual agent is then evaluated in a physical Human-Robot Interaction (pHRI) experiment. The results of the second experiment show that the virtual partner is able to perform the task without compromising the performances of the dyad and that a similar role distribution is observed in human-human and human-robot dyads. Moreover, the knowledge of the partner's nature does not seem to influence the performances. The results obtained with the virtual partner are encouraging and could be used to design kinesthetic negotiation algorithms in pHRI settings.

The main contributions of this article are: (1) supporting evidence of the possibility for humans to use the kinesthetic channel as mean of negotiation during physical Human-Human Interaction in the lightest known impedance negotiation tasks (with no virtual mass involved), (2) highlighting of the correlation between initiative and dyadic decision making, (3) design of a simple yet efficient virtual partner algorithm capable of realistic physical Human-Robot Interaction in the one-dimension tracking task used in the experimental setup. This design combines minimum jerk trajectory, switching role ability, decision criteria, and statistical parameters that are detailed in this article.

CCS Concepts: • Human-centered computing $\rightarrow$ Collaborative interaction; Haptic devices;

Additional Key Words and Phrases: Physical human-human interaction, physical human-robot interaction, haptic negotiation, comanipulation

\section{ACM Reference format:}

Lucas Roche and Ludovic Saint-Bauzel. 2022. Study of Kinesthetic Negotiation Ability in Lightweight Comanipulative Decision-making Tasks: Design and Study of a Virtual Partner based on Human-human Interaction Observation. Trans. Hum.-Robot Interact. 11, 2, Article 12 (February 2022), 23 pages.

https://doi.org/10.1145/3485753

Authors' address: L. Roche and L. Saint-Bauzel, Institut des Systèmes Intelligents et de Robotique (ISIR) - UMR7222 Sorbonne Université, CNRS, INSERM (U1150), 4 place Jussieu, Paris, 75005, France; emails: roche@isir.upmc.fr, ludovic.saint-bauzel@sorbonne-universite.fr.

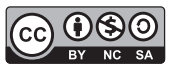

This work is licensed under a Creative Commons Attribution-NonCommercial-ShareAlike International 4.0 License.

(C) 2022 Copyright held by the owner/author(s).

2573-9522/2022/02-ART12

https://doi.org/10.1145/3485753 


\section{INTRODUCTION}

Since the early concept of cobots [1], significant progress in control, conception, and safety has brought natural human-robot interaction closer to reality. Robotic devices have evolved from rigidly programmed entities to systems that can smoothly interact with their environment and react to some amount of unknown parameters.

Robots are now more often led to work alongside humans and to cooperate with them for numerous tasks in a wide range of applications, from industry to health-care $[2,3]$. This cooperation often leads to interaction either via direct contact or via indirect contact through a jointly held object.

The ability to dynamically exchange roles during the task is a key point for efficient comanipulation $[4,5]$. Role distribution changes have effectively been observed during task realization in physical Human-Human Interaction (pHHI) settings. These role exchanges are both timevarying and dyad-dependant [6, 7]. Furthermore, depending on the task, a significant role imbalance seems to be preferred: One of the partners stays more dominant than the other [6]. A lot of different solutions have been proposed to reproduce dynamic role exchange in physical HumanRobot Interaction (pHRI), and most of them observe superior performances with dynamic role allocation than with fixed role allocation. A first approach consists in predicting human intentions to vary the amount of assistance given by a robotic partner. This prediction can be made by online estimation of the position or velocity of the human [8-10]. It can also be done by using models of the task and human motions [11] such as the homotopy switching model in Reference [12].

The amount of leadership taken by the robot can be adjusted according to the expected human behavior. Among the different approaches using the interaction forces as a means of exchanging information and negotiating role allocation online: Mortl et al. [13] use an analysis of redundancy in the dyad to allocate role according to the task. Oguz et al., Kucucyilmaz et al., Madan et al. [14-16] use measured forces as a way to negotiate the amount of assistance provided in a 2D haptic board game. Li et al. [17] used the minimization of a cost function linked to the task to modulate the robot participation, inspired by game theory. Stefanov et al. [18] introduced a theoretical role assignment framework that goes beyond the leader/follower duality, but this framework was not implemented in any real-world experiment.

The study of $\mathrm{pHHI}$ has also produced multiple results concerning the behavior of human-human dyads in comanipulative tasks. One of the first and most important results is that humans tend to perform better when operating as a dyad, which has been observed in multiple studies [19-25], although this increase in performance may depend on the type of task [26] and the presence of force feedback between the humans [27, 28]. Simply reproducing pre-recorded human trajectories, however, does not yield the same benefits as interacting as a human dyad [7, 21, 29]. There seems to be a need for real-time interaction and exchange for the dyadic benefits to take place. Interaction during $\mathrm{pHHI}$ is closely linked to the presence of some form of haptic or kinesthetic feedback. Kinesthetic feedback indeed seems to have a great influence on the success of dyadic comanipulation between humans. It has been proven to convey emotions [30] as well as an increased sense of telepresence [27, 28]. It also allows for better learning [21, 28] and performances in tracking tasks, even in cases of conflict [31]. In conclusion, many studies point at the haptic channel as an efficient means of information transmission between humans [31-35].

Even if the existence of this haptic information transmission in human dyads is a well-accepted theory, the precise mechanisms behind it are yet to be understood. Feth et al. [36] linked kinesthetic negotiation to the energy exchanges inside the dyad for the partners to negotiate between their individual motion plans. Tagaki et al. [37] found that the Central Nervous System can interpret the force signals from the haptic link and recreate the motion plan of their partner. Simulation 
with this postulate successfully reproduced the results of a previous study [21] on the benefits of dyadic interaction for performance and learning improvement. While these studies provide precious insights on the way haptic negotiation may happen, a lot has still to be understood before we can successfully replicate this ability in robots.

In most of those studies, the kinesthetic interfaces used have high impedance (mainly a great mass), so the protocols induce high interaction forces. Although high interaction forces can be desirable, the high apparent impedance of these systems limits the range of tasks that can be studied. In particular, precise and lightweight motions, which can be needed in surgery, for example, cannot be studied with these high-impedance interfaces.

The type of cooperative task is also an important parameter. The performances of the dyads will vary greatly, depending on the level of conflict induced by the task. A preliminary study [38] has shown significant difference in dyads' performances for tracking or decision-making tasks. The present article focuses primarily on decision-making and negotiation in the cooperative tasks.

All these experiments are realized on a custom made teleoperation setup using two haptic interfaces specifically designed for the study of lightweight and precise comanipulative tasks. It is conceived to provide a zero virtual mass interaction thanks to a four-channel controller architecture (a complete description of the device can be found in Reference [39]). As far as we know, this device has the smallest inertia used in study of both human-human and human-robot interaction.

The first question when trying to characterize haptic negotiation is whether humans can actually resolve a conflicting situation through kinesthesia, and how much this lightweight and precise situation affects performances in pHHI. Groten et al. in Reference [31] concluded that the presence of haptic feedback indeed enhances the precision and efficiency of human dyads in a continuous negotiating cooperative task.

The protocol used in this article uses a one degree of freedom (dof) scenario, which allows to simplify the dynamics of the task and to maintain a greater control on the parameters influencing its execution. It uses a continuous tracking task, as opposed to pointing or reaching tasks, which may be insufficient to highlight significant role adaptation in dyads [40]. It is built upon the protocol used in Reference [31], transposed to our lightweight setup to greatly reduce the influence of inertia in the interaction. The negotiation is obtained by introducing conflicting situations by giving contradicting targets to members of a dyad during a teleoperated tracking task. Users are moreover restricted to visual and kinesthetic feedback for interaction.

This experimental protocol is used for two experiments. The first one, pHHI experiment, serves two purposes. On one hand, it allows us to test our first two hypotheses: H1-that humans can use kinesthetic information for negotiation in conflicting tasks; and $\mathrm{H} 2$-that the impedance in the task does not influence this kinesthetic negotiation. On the other hand, it allows us to record sensible data on pHHI that can then be used to analyze kinesthetic communication and design a virtual partner able to perform the task alongside a human. Data from this $\mathrm{pHHI}$ experiment shows that initiative plays a great role in conflict resolution in our tracking task. Subjects that acted quicker tended to lead more of the decisions than their partners. We then design a simple virtual partner, reproducing the observed behavior and taking into account human movement properties such as minimum jerk [41] trajectories and imperfections modeled with stochastic parameters.

The two following hypotheses of this study are H3-that this virtual partner based on initiative can provide similar performances to a human partner in negotiating kinesthetic lightweight cooperative tasks; and H4-that the knowledge of their partner's nature (human or virtual) influences the subject's behavior. To explore these questions, a second experiment ( $p H R I$ experiment) is then realized to evaluate the virtual partner in interaction with humans.

Section 2 will present both experimental setups and protocol. Section 3 presents a pHHI experiment realized with the setup. Section 4 presents the virtual partner design and exposes the results 


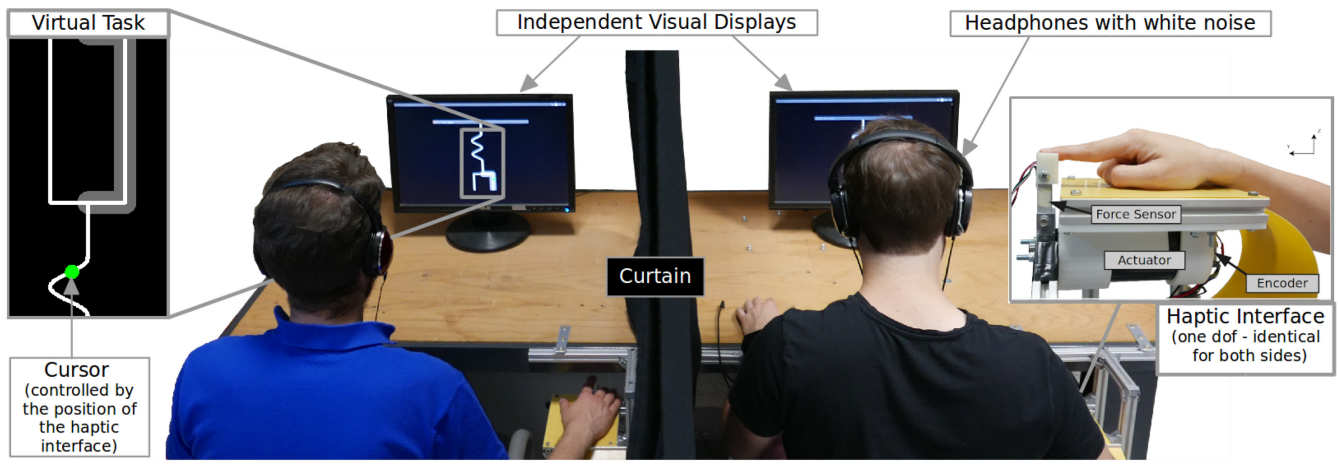

Fig. 1. Description of the experimental setup: The two participants use a one d.o.f kinesthetic interface (called $\mathrm{SEMAPHORO}^{1}$ ) to share the control over a virtual object. Visual feedback about the position of the object is given on their respective monitors as a cursor.

of the pHRI experiment evaluating the virtual partner. Last, Section 5 draws the conclusion of the present studies.

\section{EXPERIMENTS}

\subsection{Experimental Hypotheses}

Our two experiments are organized to investigate the four following hypotheses:

- H1: Humans can use kinesthetic information for negotiation in conflicting tasks.

- H2: The impedance in the task does not influence the kinesthetic negotiation.

- H3: A virtual partner based on initiative can provide similar performances to a human partner in negotiating kinesthetic lightweight cooperative tasks

- H4: The knowledge of the partner's nature (human or virtual) influences the subject's behavior.

\subsection{Experimental Apparatus}

2.2.1 The SEMAPHORO Kinesthetic Interface. ${ }^{1}$ Custom-made kinesthetic interfaces are used for the experiments presented in this article (see Figure 1). The interfaces are designed as the support of studies on human-human and human-robot interaction, especially in the context of lightweight and precise comanipulation tasks. The interfaces each have one rotational degree-of-freedom in $\mathrm{Y}$ axis that permits motion of the handle in $\mathrm{X}-\mathrm{Z}$ plane, implemented with direct drive actuation to minimize backlash and friction. Position and force sensors are used for data acquisition.

The implementation of an optimized four-channel teleoperation control scheme [42-44] allows to create a rigid virtual link between the two interfaces. When activated, this bilateral teleoperation mode allows each user to feel motions and forces applied by his/her partner through his/her own interface, as if both were holding the same object.

A full description of the interfaces design and control can be found in Roche et al. [39]. The implemented four-channel teleoperation controller can be summarized as follows:

$$
F_{m i}(t)=C_{m}\left(X_{\bar{i}}(t-T d)-X_{i}(t)\right)+C_{O F} * F_{\bar{i}}(t-T d),
$$

\footnotetext{
${ }^{1}$ Système d'Évaluation de la MAnipulation Physique HOmme-RObot - System for the Evaluation of Human-Robot Physical Manipulation.
} 
where $F_{m i}$ is the command sent to the handle interface " $i$," composed of two parts:

- A scheduled gain PD controller $\left(C_{m}\right)$ on the position difference between both handles $\left(X_{i}\right.$ and $\left.X_{\bar{i}}\right)$, defined by two parameters: $K_{\text {proportional }}=[400-1000], K_{\text {differentiate }}=[2-5]$, which vary depending on the interaction force measured between the handles.

- A proportional gain $\left(C_{O F}=1\right)$ on the interaction force between the other user $\left(F_{\bar{i}}\right)$ and his/her handle.

$T_{d}$ represents the delay in the controller loop. It is equal to one loop period, since the controller of both interfaces is run by the same process, reducing the communication delay to a minimum. The controller frequency is $2 \mathrm{kHz}$, as a consequence $T_{d}=0,5 \mathrm{~ms}$.

The controller is able to provide a high precision, as it is able to measure the handle displacement with a discrete step $0.035 \mathrm{~mm}$ width. The bandwidth of the system is $10 \mathrm{~Hz}$ with a $-3 \mathrm{db}$ cut-off. The maximum force that can be transmitted at the handle is $23 \mathrm{~N}$. The total rotational inertia of the device is $J_{h}=2.96 \times 10^{-4} \mathrm{~kg} . \mathrm{m}^{2}$, which represents a point-mass equivalent to $46 \mathrm{~g}$ at the tip of the handle. ${ }^{2}$

When no force feedback is required between users, the controller can be deactivated, allowing the interfaces to move freely from each other. In this case, each user has to manipulate the mass of its device independently, as opposed to both masses jointly, which does not induce a bias in the amount of force required to use the setup.

2.2.2 Experimental Setup. The experimental setup allows two humans to use the kinesthetic interfaces to perform various virtual tasks, alone or in cooperation (see Figure 1). Both participants are seated at a desk in front of a monitor $(19$ ", $1440 \times 900$ p). The interfaces are placed on their right side and is height-adjusted for comfort. The interfaces are manipulated with the index finger of the right hand.

The participants are separated by an opaque curtain to prevent any visual clue from their partner. They also wear audio headphones playing white noise during the experiment to prevent any auditory clue.

\subsection{Experimental Decision Types}

The experiment requires two subjects to perform a manipulative task, either alone or as a dyad.

2.3.1 Dyadic Task. The experimental task is a tracking task: A path (white line over black background) is scrolling down on their monitor, at a speed of $35 \mathrm{~mm} / \mathrm{s}$. The subjects use the kinesthetic interfaces described previously to control the position of a massless virtual object, represented on their screen as a cursor (see Figure 1). The cursor is the same for both subjects, as they share control over a single common virtual object. The subjects are asked to keep the position of the cursor as close as possible to the scrolling path. To further incite each subject to cooperate, they are told that their goal is to maximize the common performance of the dyad. Feedback about the common performance is given by the color of the cursor, which changes based on the distance between the closest path and the cursor (see Figure 2). Colors are: Green $<5 \mathrm{~mm}$ - Yellow $<10 \mathrm{~mm}$ - Red $>10 \mathrm{~mm}$.

The path is composed of a procedurally generated succession of curves, divided in two categories (see Figure 2). The BODY category is composed of sinusoidal paths of random directions but fixed duration. The purpose of these parts is to keep the subjects focused on the task between each of the studied parts. The CHOICE category is the aim of the experiment: At fixed intervals, the path splits into a fork, imposing a clear choice to be made concerning the direction that the subjects need to follow (see Figure 2). Considering that the subjects can neither see nor hear each

${ }^{2}$ For comparison, the virtual mass is $10 \mathrm{~kg}$ in Groten et al. experiments. 


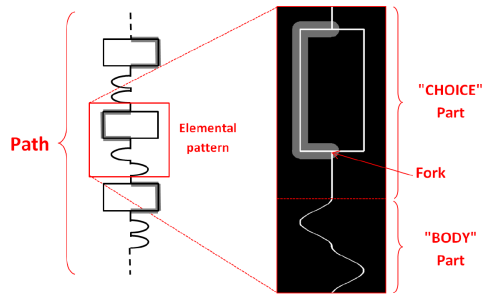

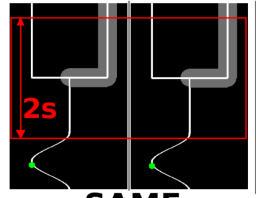

SAME

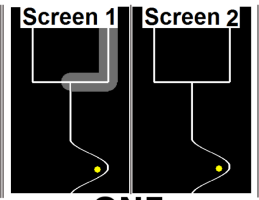

ONE

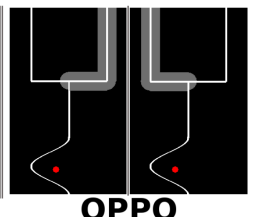

Fig. 2. Illustration of the experimental task. A pattern composed of sinusoidal parts (BODY) and a fork (CHOICE) is repeated 15 times to create each trial. The orientation of the parts is randomly generated. The CHOICE can display different decision types: SAME, ONE, and OPPO. Data about the choices is recorded from a $2 \mathrm{~s}$ timezone around the path's fork (in red on the leftward figure).

other, the only way they can come to an agreement about the direction to choose is to use either the visual feedback from the monitor or the kinesthetic feedback from the handles. While the path's structure is strictly the same for both subjects, each subject is encouraged to follow a highlighted trajectory. During the CHOICE parts, subjects receive visual information about which side they have to choose [31]; this information can differ, creating situations of agreement or conflict, distributed in three decision types. This is done by highlighting one of the two paths of the fork (see Figure 2):

- SAME: Both subjects have the same information, no conflict occurring.

- OPPO: Opposite information given to each subject, inducing a conflicting situation.

- ONE: Only one subject has the information. This condition forces the subjects to be ready to take initiative in case they are the only one having information about the path to choose. It is designed to discourage subjects from keeping a passive strategy all along the trials.

The subjects are informed about the presence of the choices and about the different information repartitions (called decision types in the rest of the article) beforehand. Each trial lasts 110 seconds, corresponding to a total of 15 choices distributed equally between SAME, ONE, and OPPO decision types. The order of decision types sequence is pseudo-random. It was generated at random before the experiments and used as "scenarios" for each trial number (e.g., scenario 1 was used for all first trials of each experimental condition, scenario 2 for each 2 nd trial...).

Statistical analysis performed on preliminary experiments showed no difference in behavior (reaction time, speed profiles) between leftward and rightward motions during the choices. We thus did not try to balance the direction of choices in the decision types, which would require the ONE decision type to be performed twice as much as the other.

2.3.2 Individual Task. In the individual conditions, the overall task is kept the same, without the negotiation component of the choices. Subjects are still asked to follow the highlighted path when they have one and to choose a direction at random in the case they do not.

\subsection{Experimental Conditions}

The two experiments presented in this article use the same experimental task. The first experiment aims at studying the behavior of human-human dyads in a comanipulative one degree of freedom task. The second experiment aims at evaluating the performances of a virtual partner in human-robot dyads on the same task. Five different experimental conditions are used across the two experiments:

- Subjects separated (ALONE): Each subject uses their own interface and has visual feedback from their monitor about their position and virtual task. Each subject can feel their 
own motions and their interface's inertia, but nothing from their partner. Both subjects perform this condition at the same time independently. The cursor positions are also separated: $x_{\text {cursor } . i}=x_{i}$ (where $i \in\{1,2\}$ ). Orientation sensors drive the position of the cursor, force sensors are recorded for comparative analysis.

- Haptic-Feedback-from-Object (HFO): In this condition, the two handles are kept free to move independently. Each subject can feel their own motions and their interface's inertia, but nothing from their partner. Each subject contributes equally to the task: The position of the cursor is identical on each screen and computed as the average of each handle positions: $x_{\text {cursor }}=\left(x_{1}+x_{2}\right) / 2$. Hence, subjects can infer the input of their partner by interpreting the movements of the cursor that are not caused by their own handle's movements.

- Haptic-Feedback-from-Object-and-Partner (HFOP): As in HFO, the subjects contribute equally to the cursor position. Haptic feedback is added between the interfaces through the teleoperation controller, which simulates a rigid connection between the interfaces. The subjects are then able to feel the efforts applied on the interfaces by both them and their partner. The position of the cursor on the screen is similar to previous condition: $x_{\text {cursor }}=$ $\left(x_{1}+x_{2}\right) / 2$.

- Hidden Virtual Partner (HVP): The subjects believe they are doing the task together, but they are actually performing their task independently, each paired with their own virtual partner. The subjects have visual feedback concerning their own task and virtual object on their monitor and can feel the kinesthetic feedback from the virtual partner. The position of each cursor is computed from the virtual partner position and human position: $x_{\text {cursor. } i}=$ $\left(x_{i}+x_{V P i}\right) / 2$ (where $i \in\{1,2\}$ and $V P i$ is the Virtual Partner replacing User $\bar{i}$ ).

- Known Virtual Partner (KVP): This condition is the same as HVP, with the difference that the subjects are told beforehand that their partner is a virtual agent. This condition is used to compare the behavior of the human subjects depending on their a priori about their partner. The cursor position computation is similar to HVP condition.

\subsection{Metrics}

2.5.1 Root-Mean-Squared Error-RMS. When studying physical interaction, the first criterion used for evaluation is generally the performance in the realization of the task. In the case of a tracking task, this performance is linked to the precision of the tracking. The tracking error is calculated using RMS error (chosen over simple position error because it amplifies the influence of large errors on the result):

$$
R M S=\sqrt{\frac{\sum_{k=1}^{N}\left(x_{t, k}-x_{o, k}\right)^{2}}{N}},
$$

where $x_{t, k}$ and $x_{o, k}$ are, respectively, the target position and the virtual object position at time step k. $N$ represents the number of samples evaluated during the "CHOICE" part. Performance is then obtained by comparing the RMS error for a choice to the maximum RMS obtained on the whole set of trials in every condition $R M S_{\text {max }}$ :

$$
\text { Performance }=1-\frac{R M S}{R M S_{\max }} .
$$

This Performance indicator is preferred over RMS error for clarity: The better the results, the greater the Performance. It can be noticed that if an abnormally high RMS occurs in the result, this single point of measure can skew the values of Performance for the whole set. To avoid this, results are filtered and values of RMS that are above $|R M S|+3.5 * \sigma$ are removed, corresponding to the $0.05 \%$ most extreme cases. 
2.5.2 Mean Absolute Power - MAP. The second aspect of physical interaction that needs to be studied is the physical efforts exerted on and by the interfaces, as well as the interaction force between the participants. The metric used combines both forces and motions to address the physical cost of movements, which leads to energy- or power-based measures. The MAP criterion introduced by Groten et al. in Reference [31] is chosen for this measure. It is defined as the sum of absolute values of the power flows from the subjects to their interfaces:

$$
M A P=M A P_{1}+M A P_{2}=\frac{1}{N} \sum_{k=1}^{n}\left|P_{1, k}\right|+\frac{1}{N} \sum_{k=1}^{n}\left|P_{2, k}\right|,
$$

where $P_{1, k}=x_{o, k} \cdot F_{1, k}$ and $P_{2, k}=x_{o, k} \cdot F_{2, k}$ are the mean instantaneous power at the respective kinesthetic interfaces at time step $\mathrm{k}$ (with $x_{o, k}$ the velocity of the virtual object and $F_{x, k}$ the force applied on interface $\mathrm{x}$ ). $N$ is similar to RMS equation.

2.5.3 Dominance - DOM. In OPPO decision type condition, the dyad has to choose between the two contradictory options that are presented. Since the cursor is common to the two partners, only one of them can "win" i.e., reach his/her highlighted side. The partner winning will be defined as the leader for the choice, and his/her partner as the follower. The "Dominance" of a participant is defined as his/her propensity to Lead in the conflicting choices, i.e., the percentage of trials in OPPO condition where the subjects impose their choice to their partner.

$$
D O M_{s}=\frac{n_{s, \text { win }, O P P O}}{n_{O P P O}},
$$

where $s \in\{1,2\}$ represents the subject, $n_{O P P O}$ is the number of trials with OPPO choice, and $n_{s, \text { win, } O P P O}$ is the number of trials where the subject has won the negotiation. Each human member of each dyad is classified as a Leader or Follower, depending on his/her dominance across the experience. Levels of dominance are investigated for all experimental conditions. When compared to a virtual partner, the human keeps his Leader/Follower from the HFOP experimental condition.

\subsection{Statistical Analysis}

In the results section, different statistical tools will be used. For Learning Effect a one-way ANOVA is performed. For simple null-hypothesis evaluation, $t$-tests with significance factor lower than $5 \%$ $(p<0.05)$ will be considered. And for multiple comparisons, the statistical analysis is here performed with repeated measures two-ways ANOVA and post hoc analysis with two-tailed Student's t-tests with Bonferonni correction. The choice of these statistical analysis tools is done because they are the most used in the literature of pHHI and pHRI. Indeed, when comparing individuals to dyads, statistical analysis of the data can be challenging: Subjects cannot be expected to behave the same in solo trials and in dyadic ones, thus a repeated measures design does not really fit. However, a between-subjects design would assume that individuals and dyads are independent entities, which is similarly problematic. In the literature concerning individual-dyads comparison for pHHI tasks, Reed et al. [19] and Che et al. [26] used paired sample t-tests. Feth et al. [36], Van der Wels et al. [34], and Mireles et al. [45] used repeated measures ANOVA. Other articles used ANOVA without precision of the design considered [21, 24]. Repeated measures ANOVA has been chosen in this article for MAP and performance evaluations as the method that seems the most used. Results of ANOVA in the next sections are given with the following form: ANOVA (F-value, p-value, omega-squared effect-size value), t-tests (Bonferonni corrected p-value, Cohen's $d$ coefficient for effect-size). 


\subsection{Ethics}

This research was reviewed and approved by the institutional ethics committee CERES of University Paris Descartes (2015-11-10). Informed consent was obtained from each participant.

\section{HUMAN-HUMAN KINESTHETIC NEGOTIATION ABILITY EVALUATION}

\subsection{Protocol}

Three different experimental conditions are tested in this experiment to study the influence of the presence of kinesthetic feedback on the performances in a comanipulative task: ALONE, HFO, and HFOP.

Each dyad starts the experiment with a block of two trials in ALONE condition to familiarize with the interface and its control; this first block is not kept for the following analysis. They continue with the first experimental block, consisting of two trials in either HFOP or HFO condition. A trial in ALONE condition is tested afterwards, followed by two trials in the condition that is not tested in the first block between HFOP and HFO. The experiment ends with a final trial in ALONE condition. These two possibilities are presented below:

\begin{tabular}{|c|c|c|c|c|}
\hline$A L O N E(\times 2)$ & $\begin{array}{c}H F O(\times 2) \\
\bar{H} \overline{F O} \bar{P}(\overline{\times}) \overline{2}\end{array}$ & $A L O N E(\times 1)$ & $\begin{array}{l}H F O P(\times 2) \\
\overline{H F} \overline{(\times 2)}\end{array}$ & $A L O N E(\times 1)$ \\
\hline
\end{tabular}

The order between HFO and HFOP is pseudo-randomized, and the ALONE condition is always tested between these two to prevent learning effects from one condition to another. The last trial in ALONE condition is used to check for potential learning effects during the experiment (compared to the training block). A 40-second pause is respected between each trial. At the beginning of the experiment, the subjects are explained the rationale of the setup and told about the different choices in the task. They are also told that three different experimental conditions are tested: they can either perform the task alone (ALONE), cooperate through co-manipulation (HFOP), or cooperate with visual feedback only (HFO).

The study involved 30 participants (15 males and 15 females) distributed in 15 dyads (6 MaleMale, 6 Female-Female, 3 Mixed). Participants' average age was 21.3 (std 4.3 y). All participants were right-handed and had no previous knowledge of the experiment or the experimental setup. Each dyad provided data for every experimental condition.

\subsection{Results}

The results of the first experiment are explained in this section. The independent variables are Experimental Condition (ALONE, HFOP, HFO) and Decision Type (SAME, ONE, OPPO). The averages in efforts (MAP), Performances, and dominance are studied for each combination of Experimental Condition and Decision Type. Data is analyzed over a two-second window around the Choice (see Figure 2), where the potential for kinesthetic communication is the greatest. The parts of the trials outside this window mainly consist in a pure tracking task, which is not the main focus of this article. A more detailed study on tracking and negotiation tasks can be seen in Reference [46]. Data from all trials are used except for the first block in ALONE condition, for a total of two trials (30 choices) for each condition. Since the experimental protocol insists on completing the motions as a priority, we did not observe motions that did not finish at the end of the FORK parts $(3 \mathrm{~s}$ duration). We did, however, observe some motions that did not end within 2 seconds of the FORK part (less than $2 \%$ ).

3.2.1 Learning Effect. The experimental design used for this experiment is not entirely counterbalanced: All dyad conditions are tested after an individual trial, but not always after the 
Table 1. Influence of the Decision Type over Mean Absolute Power (MAP) Criterion, Depending on the Experimental Condition

\begin{tabular}{|l|ccccc|}
\hline Condition & SAME & vs. & ONE & vs. & OPPO \\
\hline HFOP & SAME & $<^{*}$ & ONE & $<^{* *}$ & OPPO \\
HFO & SAME & $<^{* *}$ & ONE & $<^{* *}$ & $O P P O$ \\
ALONE & SAME & $\sim$ & ONE & $\sim$ & OPPO \\
\hline
\end{tabular}

Decision Types are SAME (same target for both subjects), ONE (only one subject has a target), and OPPO (different targets for each subject). Experimental conditions are ALONE (subjects do the task independently), HFO (subjects do the task as dyads, with visual feedback only), and HFOP (dyads with visual and kinesthetic feedback).

Table 2. Influence of the Experimental Condition over MAP Criterion, Depending on the Decision Type

\begin{tabular}{|l|ccccc|}
\hline Type & ALONE & vs. & HFOP & vs. & HFO \\
\hline SAME & ALONE & $\sim$ & $H F O$ & $<^{* *}$ & HFOP \\
ONE & ALONE & $<^{* *}$ & $H F O$ & $<^{* *}$ & HFOP \\
OPPO & ALONE & $<^{* *}$ & $H F O$ & $<^{* *}$ & HFOP \\
\hline
\end{tabular}

same number of total trials. This poses a risk for the statistical analysis if a learning effect is observed between the different trials. A One-way repeated measure ANOVA does not show any significant effect of the trial number on the performances in any experimental condition $\left(\right.$ ALONE: $F(3,60)=1.38, p=0.25, \omega^{2}=0.004$; HFO: $F(1,30)=0.17, p=0.73, \omega^{2}=0.001$; HFOP: $\left.F(1,30)=2.06, p=0.15, \omega^{2}=0.013\right)$. Moreover, performance is not significantly affected by the order of the experimental condition: Student's t-tests, HFO first vs. HFO second $(p=0.33, d=0.01)$; HFOP first vs. HFOP second ( $p=0.53, d=0.04)$.

3.2.2 Mean Absolute Power (MAP). There is a significant interaction between decision type and experimental condition $\left(F(4,416)=33.94, p<0.001, \omega^{2}=0.088\right)$, post hoc analysis is thus performed to observe the MAP variation in each (decision type)*(experimental condition) pair. The MAP values for each Decision Type and Experimental Conditions are presented in Figure 3. Planned comparisons have been performed to study differences in MAP; they are described in Tables 1 and 2. Details of the t-tests are omitted for clarity; significant differences with a p-value less than 0.05 are signaled with a $\left(^{*}\right)$; p-values less than 0.001 are signaled with a $\left(^{* *}\right)$.

3.2.3 Performances. There is a significant interaction between decision type and experimental condition $\left(F(4,416)=17.38, p<0.001, \omega^{2}=0.11\right)$, post hoc analysis is thus performed to observe the performance variation in each (decision type) * (experimental condition) pair. For the experimental condition, post hoc analysis reveals that the performances were highest in the ALONE condition, followed by the HFOP condition, with HFO condition leading to the worst performances except in SAME trials where HFO and HFOP lead to similar performances. The performances are presented in Figure 4. Planned comparisons have been performed to study differences in performance; they are described in Tables 3 and 4. Details of the t-tests are omitted for clarity, significant differences with a p-value less than 0.05 are signaled with a $\left(^{*}\right)$, and p-values less than 0.001 are signaled with a $\left({ }^{* *}\right) . \sim$ character is shown when there is no statistical difference. 


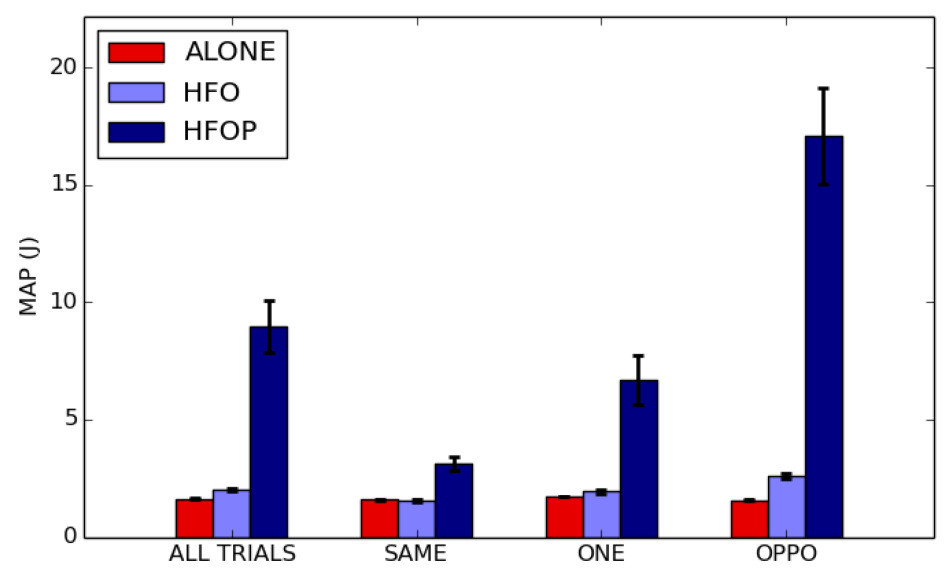

Fig. 3. MAP parameter results for the $\mathrm{pHHI}$ experiment. (Error bars represent standard errors of the distributions.)

Table 3. Influence of the Decision Type over Performance, Depending on Experimental Condition

\begin{tabular}{|l|ccccc|}
\hline Condition & SAME & vs. & ONE & vs. & OPPO \\
\hline HFOP & SAME & $\sim$ & $O N E$ & $>^{*}$ & OPPO \\
HFO & SAME & $>^{* *}$ & $O N E$ & $>^{* *}$ & $O P P O$ \\
ALONE & SAME & $\sim$ & $O N E$ & $\sim$ & $O P P O$ \\
\hline
\end{tabular}

Decision Types are SAME (same target for both subjects), ONE (only one subject has a target), and OPPO (different targets for each subject). Experimental conditions are ALONE (subjects do the task independently), HFO (subjects do the task as dyads, with visual feedback only), and HFOP (dyads with visual and kinesthetic feedback).

Table 4. Influence of the Experimental Condition over Performance, Depending on Decision Type

\begin{tabular}{|l|ccccc}
\hline Dec. Type & HFO & vs. & HFOP & vs. & ALONE \\
\hline SAME & $H F O$ & $\sim$ & HFOP & $<^{* *}$ & ALONE \\
ONE & $H F O$ & $<^{*}$ & $H F O P$ & $<^{* *}$ & $A L O N E$ \\
OPPO & $H F O$ & $<^{* *}$ & $H F O P$ & $<^{* *}$ & ALONE \\
\hline
\end{tabular}

3.2.4 Dominance. The Leader won $84.6 \%( \pm 19 \%)$ of the conflicting choices in the HFOP condition. The difference between Leader and Follower dominance was statistically significant $(p<$ $0.001, d=3.67)$. The Leader won $76.5 \%( \pm 28 \%)$ of the conflicting choices in the HFO condition. The difference between Leader and Follower dominance was statistically significant $(p<0.001, d=$ 2.27). The difference of dominance between the HFOP and HFO conditions was not statistically significant for both the Leader and the Follower.

3.2.5 Negotiation Analysis. When observing the negotiation phase during an OPPO condition, the emerging behavior has been observed as presented in Figure 5. As one can see, there is a discrepancy between the leader position and the follower position. This is mainly due to the pulling action of the leader on the follower. This pulling effect is what let us assume that this criteria is an image of the initiative of the leading partner. As one can see in the given figure, this is a situation where the hesitation is easy to see. While looking in detail into this example, it starts with subject 


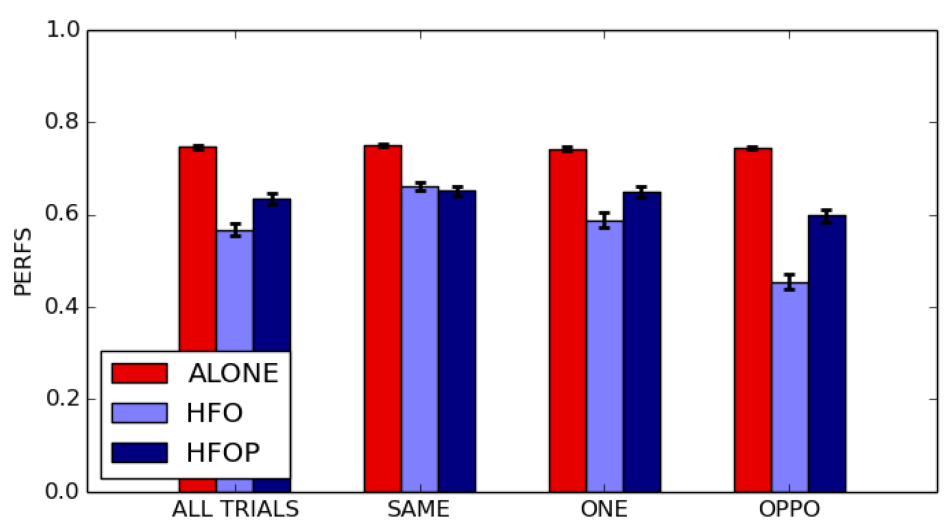

Fig. 4. Performance parameter results for the $\mathrm{pHHI}$ experiment. (Error bars represent standard errors of the distributions.)

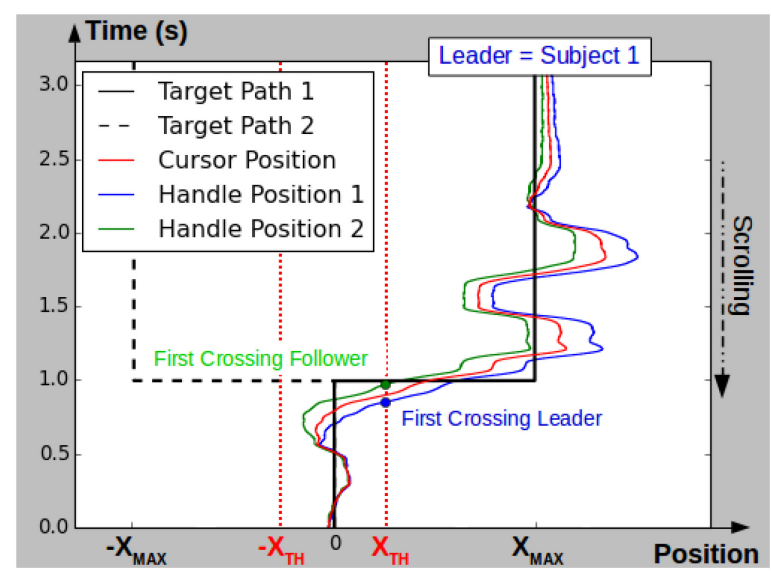

Fig. 5. Emerging behavior during OPPO condition of dyads. Position difference between subjects is exaggerated for clarity.

one leading to the right, then subject 2 takes the lead, and finally subject 1 obtains his/her desired position. The decision criteria is using this pulling effect as a smart way to identify which one is leader and which mode the virtual partner must follow. The choice of the threshold is strategic. As one can see, it could have been wrong in this example if it was smaller. The $X_{\text {cursorthreshold }}\left(X_{T H}\right)$ is chosen as the best tradeoff between being close to the center and having a good prediction accuracy. With a $X_{\text {cursorthreshold }}=35 \%$ of the final position $\left(X_{M A X}\right)$, the criteria is able to guess $95.29 \%$ of the final positions.

\subsection{Discussion}

The first experiment aims at illustrating differences in performances and interaction force brought by the addition of tactile feedback in physical Human-Human Interaction (pHHI). Thirty participants (15 dyads) use a one degree of freedom dual kinesthetic interface to realize a onedimensional tracking task.

The results show that the best performances are obtained in the ALONE condition. While these results seem to be in contradiction with the common finding that dyads outperform individuals, 
it can be explained by the nature of the task. Most of the studies concerning pHHI use tasks that only involve coordination in basic pointing or target tracking and do not require the subjects to negotiate a choice. The results presented here concern the time period around the decisionmaking parts of the task. It is thus natural that dyads, who need to come to an agreement about the direction to choose, are outperformed by individuals, who do not have this cognitive burden to handle. Interestingly, this observation holds true even in the SAME condition, in which no conflict between subjects should arise, since they have the same indications. However, since the subjects cannot know in advance in which decision type they are, we can assume that they still need to consider the possibility of a conflict, thus hindering their performances.

Performances are significantly degraded in the HFOP condition compared to ALONE, with the implementation of a necessity to handle conflicting situations. The performances are even worse in the HFO condition. The superior performances obtained in HFOP compared to HFO can be explained by the superior quantity of information available to the subject to negotiate the conflicting situation through the kinesthetic channel.

This observation can be corroborated by the fact that the MAP criterion is significantly higher in HFOP condition than in HFO, meaning that more energy was expended during the task. Since the energy necessary to accomplish the task is the same for both conditions, without excluding additional communication processes, which may not be represented in the average effort measure used here, we can observe that the haptic channel takes advantage of this additional energy to communicate with their partner. The MAP criterion is the highest in the OPPO trials, followed by the ONE trials and last the SAME trials. These results show a link between the energy consumption and the necessity for negotiation. Indeed, the SAME decision type choices should not lead to conflict and therefore show the lowest MAP criterion. The ONE decision type choices need some negotiation to take place; since only one participant has information about the target, the other one needs to extract information about this target, which could be done through the kinesthetic communication channel. The OPPO decision type choices are by definition conflicting and show the highest energy expenditure, in agreement with the proposed hypothesis.

The main results, namely, that the addition of kinesthetic feedback in comanipulative tasks leads to greater efforts and performances, are consistent with the work done by Groten et al. [31], while obtained with a different experimental apparatus. This reinforces the hypothesis that humans can indeed negotiate via a kinesthetic channel (H1). Moreover, this negotiation seems to be present in tasks involving varying impedance and forces (H2). In overall analysis, the similarities and differences (which condition is greater than the other) remains consistent in our observation compared to literature.

However some differences in results are found between this study and Groten et al. [31]: For the MAP criterion, a greater difference between the HFO and HFOP conditions is found in the present study, as well as a significant difference between the SAME and ONE decision types. For the performance criterion, the present study found significant differences between decision types in HFOP while none were found in Reference [31]. These differences could be explained by the change of scale in the experimental apparatus, leading to a different sharing between the efforts used for the task and those used for negotiation.

Overall, the decision-making appears heavily in favor of one of the two participants in most of the experiments. In almost every dyad, one of the two participants acted as a "Leader" and decided the direction in most of the conflicting situations; the other participant acting like a "Follower." This dominance discrepancy is in agreement with previous results [6] and was more pronounced in the HFOP condition than the HFO condition, which could be explained once again by the higher amount of information available for negotiation, helping the leader cement his role more easily. 


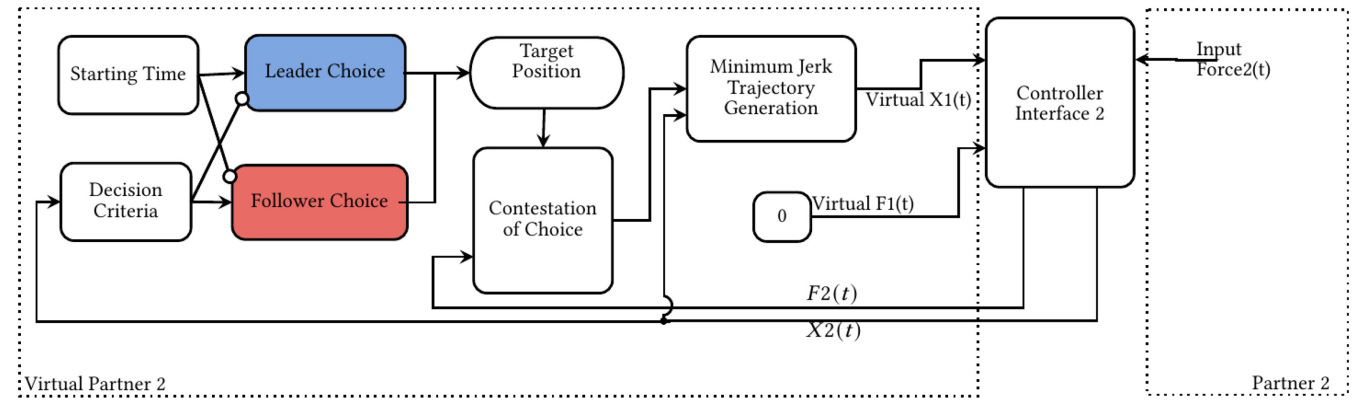

Fig. 6. Virtual partner algorithm in Choice section: VP2 is a Virtual Partner interacting with the Human Partner number 2.

\section{2ND EXPERIMENT - VIRTUAL PARTNER KINESTHETIC NEGOTIATION ABILITY EVALUATION}

\subsection{Virtual Partner Design}

Based on results from the human-human interaction experiment, a virtual partner is proposed. The design approach is to mimic the human behavior and its properties. This section will detail the partner algorithm with an explanation of each part. The main goal of this virtual partner is to avoid any obvious haptic clue of the nature of the partner. The implementation of a minimum jerk motion gives natural, human-like trajectories. The use of stochastic parameters tuned with observed data allows to simulate errors in the trajectories. The ability to switch role by a contestation of choice strategy also comes from observations during $\mathrm{pHHI}$ experiments, both ours and in the literature.

In a preliminary work presented in Reference [47], a first implementation of a virtual partner was done on a previous setup that was lacking force sensors and thus did not use $4 \mathrm{C}$ teleoperation control. In this article, the complete virtual partner algorithm pipeline is presented, along with its parameters and their stochastic properties, as well as its connections with the $4 \mathrm{C}$ controller.

4.1.1 Virtual Partner Behavior. The Virtual Partner (see Figure 6) is designed to model human behavior. Therefore, the algorithm only has access to information that would be otherwise available to a human subject: (a) The target trajectory; (b) The position of its handle (simulated); (c) The position of the cursor on the monitor; (d) The effort transmitted through the handle. Indirectly, the algorithm can also determine the position of its partner's handle (through the position of the cursor and its own handle).

In the BODY parts, the algorithm follows the path. The path-following trajectory generated by the virtual agents (VP1 and VP2, each human being coupled with his own VP) are not perfect by design with regard to the execution of the task (precise tracking of the target). This is done intentionally so the performances of the virtual agent do not influence the behavior of the human partner during the task. The virtual partner follows the trajectory with a PD controller acting on the error between its position and the tracked one. The gains of this controller $\left(C_{t}\right)$ are chosen to induce a small amount of (varying) error (cf. Table 5). It leads to an imperfect final position near the desired trajectory. Since this position is unpredictable, it avoids providing unwanted clues betraying the robotic nature of the partner.

The behavior of the Virtual Partner when confronted with a CHOICE is presented in Figure 6. The algorithm draws a starting time from a Gaussian distribution. This starting time represents the time at which the Virtual Partner plans to start its motion, corresponding to the reaction time observed in human subjects. When the starting time $\left(t_{\text {start }}\right)$ is reached and Decision Criteria (First Crossing) is not verified yet, the virtual partner is triggered to be in "Leader Mode," activating the 
"Leader Choice" Component and inhibiting the "Follower Choice." The trajectory is computed from the current measured position and time. The algorithm enters "Follower mode" when the decision criteria is reached before the Starting Time, meaning that the human took the initiative before the virtual partner. In this case, the algorithm lets the human lead, activating "Follower Choice." This activation inhibits the "Leader Choice." Each component "Leader Choice" or "Follower Choice" provide a target destination $X_{f}$ (left or right branch of the fork). This target position is used to compute the trajectory that is followed by the virtual partner. If the Virtual Partner is in Leader mode, then $X_{f}$ corresponds to the Virtual Partner's target in the task. In Follower mode, $X_{f}$ corresponds to the human partner's target (inferred from the side he/she is moving toward). In the ONE decision type trials where the virtual partner does not have information on the privileged direction, the distribution used to generate $t_{\text {start }}$ is slightly delayed compared to the baseline to reflect a longer decision time.

The target position can be contested at any time by the "Contestation of Choice" component. This component implements an ability to contest the choice. The algorithm measures the part of the interaction force between the partners that is directed toward a change of trajectory (negative when the virtual object is currently on the right, and positive when the virtual object is on the left). As proposed by Reference [14], a force threshold $F_{\text {threshold }}$ is chosen according to the experimental data. When this interaction force exceeds the force threshold $\left(\frac{-\operatorname{sign}\left(x_{i}\right) \cdot F_{\text {human }}}{F_{\text {threshold }}}>1\right)$ for a duration of $\Delta_{t h}$ (defined from the average human reaction time) the virtual partner switches to following mode and the target is set to the other target destination. When the target destination has been changed a new trajectory is computed. This change in trajectory can arise multiple times if the conditions are met.

The trajectory generator is based on a minimum-jerk model starting at the current position of the virtual partner and ending at the target. The followed trajectory is generated from the minimum-jerk equation proposed by Flash et al. [41]:

$$
X(t)=X_{o}+\left(X_{f}-X_{o}\right) *\left(10 \tau^{3}-15 \tau^{4}+6 \tau^{5}\right),
$$

where $\tau=\left(t-t_{\text {start }}\right) /$ Duration and $X_{o}$ and $X_{f}$ are, respectively, the origin and the target positions on the fork. Where $X_{f} \in\left\{-X_{M A X}, X_{M A X}\right\}$. $X_{M A X}\left(-X_{M A X}\right)$ represents the right (left) part of the fork.

This minimal jerk equation generates a bell-shaped speed and consequently a smooth position trajectory; it is generated from its current position to the target position based on the choice it has to make.

4.1.2 Decision Criteria: The First Crossing Parameter (1C). The dyads start the CHOICE part with a position that is close to zero (see Figure 2). The First Crossing parameter is defined as the sign of their position when exiting the chosen interval $\left[-X_{\text {cursorthreshold }}, X_{\text {cursorthreshold }}\right]$. Negative values mean the subjects went to the left, and positive to the right. The analysis performed to find the First Crossing has two principal parameters: the time at which the analysis starts and the size of the threshold $X_{\text {cursorthreshold. }}$. Analysis of the data from the $\mathrm{pHHI}$ experiment shows that the optimal set of parameters for the task is a threshold size equivalent to $35 \%$ of the total target motion, coupled with an analysis starting at 0.2 second. These chosen parameters lead to a prediction success rate of $95.29 \%$ after $1.5 \mathrm{~s}$ of the analyzed timezone. This First Crossing criteria is also defining which partner crosses the boundary $\left(X_{\text {cursorthreshold }}\right)$ first. The partner is identified because each handle has a slightly different position due to the $4 \mathrm{C}$ teleoperation controller delay $\left(T_{d}\right.$ in Equation (1)). So the motion of one user in one direction is pulling the other. It is seen with a small offset in position that is caused at least by the delay and also by all the dynamics of the controller. This front position defines the leader of the motion. It could be seen as an image of the initiative taken during a dyadic kinesthetic exchange. 
Table 5. Description of the Parameters of the VP Algorithm

\begin{tabular}{|c||c|c|}
\hline Experimental Setup & Parameter & Value \\
\hline \hline Virtual Partner components & & $\mathcal{N}(0.886,0.160)^{*}$ \\
\hline Starting Time & $t_{\text {start }}$ & $35 \%{ }^{*} X_{M A X}$ \\
First Crossing (1C) & $X_{\text {cursorthreshold }}$ & $(1 \mathrm{~N}, 0.2 \mathrm{~s})$ \\
Contestation of Choice & $\left(F_{\text {threshold }}, \Delta_{\text {th }}\right)$ & $\mathcal{N}(0.55,0.14)$ \\
Minimum Jerk Trajectory Generator & Duration & $(50,1,1)^{* *}$ \\
Tracking controller $\left(C_{t}\right)$ & $\left(K_{\text {proportional }}, K_{\text {differentiate }}\right)$ & ( \\
\hline
\end{tabular}

*Except for ONE Condition, it is $\mathcal{N}(1.1,0.1)$.

${ }^{* *}$ In ALONE $\left(K_{\text {proportional }}, K_{\text {differentiate }}\right)=(110,1)$.

4.1.3 Virtual Partner Parameters. Table 5 presents the different parameters used to define the VP behavior, which are tuned based on human analysis, and their link to Figure 6 .

This Virtual Partner Algorithm is combining a contesting ability, a minimum jerk generator, stochastic parameters, and controller gains based on Human Human Interaction observation. It is designed to be simple and it is expected to simulate a partner that brings similar results than a human in kinesthetic negotiation. If so, then we can assume that this virtual partner can be considered as an understandable computational model of the kinesthetic decision between humans, and thus that initiative taking is a crucial point of negotiation in $\mathrm{pHHI}$.

\subsection{Protocol}

The second experiment uses four experimental conditions, designed to evaluate the performances of the virtual partner and the influence of a priori knowledge on the nature of this partner. Preliminary results have already been presented [47]. This previous study used another kinesthetic interface with limited capabilities and no force sensors. Tests using the new interfaces are thus conducted to assess the validity of the previous results (H3). Moreover, a new experimental condition is added to differentiate the cases where the participants were aware or not of the nature of their partner (H4). The task and experimental setup are similar to those exposed in the previous section, with only the experimental conditions changing.

The participants are the same as in the previous experiment. The experimental conditions tested are ALONE, HFOP, HVP, and KVP. Each experiment starts with a block of two trials in ALONE condition to familiarize with the interface and its control. This first block is not kept for the following analysis. The following trials are divided into three blocks of two trials (HFOP, HVP, KVP), each separated by one trial in ALONE condition:

\begin{tabular}{|c|c|c|c|c|c|}
\hline $\begin{array}{c}A L O N E \\
(\times 2)\end{array}$ & $\overline{H F O P}(\times 2)$ & $\begin{array}{c}A L O N E \\
\bar{H} \bar{P}(\overline{\times} 2)\end{array}$ & $\begin{array}{c}H V P(\times 1) \\
(\times 1)\end{array} \overline{\bar{H} O \bar{P}(\times \overline{\times})}$ & $\begin{array}{c}A L O N E \\
(\times 1)\end{array}$ & $\begin{array}{c}K V P \\
(\times 2)\end{array}$ \\
\hline
\end{tabular}

The order between HFOP and HVP is pseudo-randomized, and the ALONE condition is tested between these two to prevent learning effects from one condition to another. Since the KVP condition relies on informing the participants about the presence of the virtual partner, it is always tested last to avoid a potential influence on their behavior during the other conditions.

\subsection{Results}

The results of the second experiment are exposed in this section. The independent variables are Experimental Condition (ALONE, HFOP, HVP, KVP) and Decision Type (SAME, ONE, OPPO). The means in efforts (MAP), performances, and dominance are studied for each combination of 
Table 6. Influence of the Decision Type over Mean Absolute Power (MAP)

\begin{tabular}{|l|lllll|}
\hline Condition & SAME & vs. & ONE & vs. & OPPO \\
\hline HFOP & SAME & $<^{*}$ & ONE & $<^{* *}$ & OPPO \\
HVP & SAME & $<^{* *}$ & ONE & $<^{* *}$ & $O P P O$ \\
KVP & SAME & $<^{* *}$ & $O N E$ & $<^{* *}$ & $O P P O$ \\
ALONE & SAME & $\sim$ & $O N E$ & $\sim$ & $O P P O$ \\
\hline
\end{tabular}

Decision Types are SAME (same target for both subjects), ONE (only one subject has a target), and OPPO (different targets for each subject). Experimental conditions are ALONE (subjects do the task independently), HFOP (humanhuman dyads with visual and kinesthetic feedback), HVP (human-virtual partner dyads where the human thinks it is HFOP), and KVP (human-virtual partner dyads where the human knows he is paired with a virtual agent).

Table 7. Influence of the Experimental Condition over MAP

\begin{tabular}{|l|ccccccc|}
\hline Dec. Type & ALONE & vs. & HFOP & vs. & HVP & vs. & KVP \\
\hline SAME & ALONE & $<^{* *}$ & HFOP & $<^{*}$ & $H V P$ & $<^{* *}$ & $K V P$ \\
ONE & ALONE & $<^{* *}$ & $(H F O P$ & $\sim$ & $H V P)$ & $<^{* *}$ & $K V P$ \\
OPPO & ALONE & $<^{* *}$ & HVP & $<^{* *}$ & $($ KVP & $\sim$ & HFOP $)$ \\
\hline
\end{tabular}

Experimental Condition and Decision Type. The statistical analysis method and the presentation of the results are the same as in Section 3.2.

4.3.1 Learning Effect. The experimental design used for this experiment is not entirely counterbalanced: All dyad conditions are tested after an individual trial, but not always after the same number of total trials. This poses a risk for the statistical analysis if a learning effect is observed between the different trials. A one-way repeated measure ANOVA does not show any significant effect of the trial number on the performances in any experimental condition (ALONE: $F(3,60)=1.38, p=0.25, \omega^{2}=0.004$; HFOP: $F(1,30)=2.06, p=0.15, \omega^{2}=0.013$; HVP: $F(1,30)=1.02, p=0.36, \omega^{2}=0.0001$; KVP: $\left.F(1,30)=0.27, p=0.61, \omega^{2}=0.004\right)$. Moreover, performance is not significantly affected by the order of the experimental condition: Student's t-tests, HFOP first vs. HVP first (HFOP: $p=0.45, d=0.021$; HVP: $p=0.62, d=0.015$ ).

4.3.2 Mean Absolute Power (MAP). There is a significant interaction between decision type and experimental condition $\left(F(4,696)=14.04, p<0.001, \omega^{2}=0.084\right)$; post hoc analysis is thus performed to observe the performance variation in each (decision type)*(experimental condition) pair. The analysis reveals that there is no influence of the Decision Type over the MAP criterion while being in ALONE condition, and that the differences between HFOP, HVP, and KVP conditions are mainly significant in the OPPO decision type. Planned comparisons have been performed to study differences in efforts exchanges; they are described in Tables 6 and 7. Details of the t-tests are omitted for clarity, significant differences with a p-value less than 0.05 are signaled with a $\left({ }^{*}\right)$, and p-values less than 0.001 are signaled with a $\left(^{* *}\right) . \sim$ character is shown when there is no statistical difference. The MAP results are presented in Figure 7.

4.3.3 Performances. There is a significant interaction between decision type and experimental condition $\left(F(6,696)=17.55, p<0.001, \omega^{2}=0.023\right)$; post hoc analysis is thus performed to observe the performance variation in each (decision type) * (experimental condition) pair. Planned 


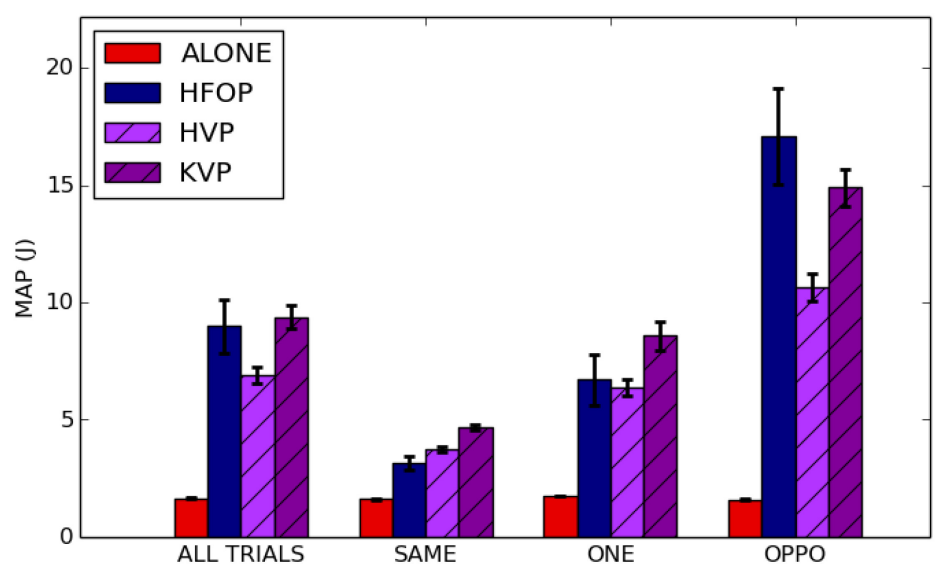

Fig. 7. MAP parameter results for the pHRI experiment. (Error bars represent standard errors of the distributions.)

Table 8. Influence of the Decision Type over Performance, Depending on Experimental Condition

\begin{tabular}{|l|ccccc|}
\hline Condition & SAME & vs. & ONE & vs. & OPPO \\
\hline HFOP & $(S A M E$ & $\sim$ & $O N E)$ & $>^{*}$ & OPPO \\
HVP & SAME & $>^{* *}$ & $O N E$ & $>^{* *}$ & OPPO \\
KVP & SAME & $>^{* *}$ & ONE & $>^{* *}$ & OPPO \\
ALONE & SAME & $\sim$ & $O N E$ & $\sim$ & $O P P O$ \\
\hline
\end{tabular}

Decision Types are SAME (same target for both subjects), ONE (only one subject has a target), and OPPO (different targets for each subject). Experimental conditions are ALONE (subjects do the task independently), HFOP (human-human dyads with visual and kinesthetic feedback), HVP (human-virtual partner dyads where the human thinks it is HFOP), and KVP (human-

virtual partner dyads where the human knows he is paired with a virtual agent).

Table 9. Influence of the Experimental Condition over Performance, Depending on Decision Type

\begin{tabular}{|l|lllllll|}
\hline Dec. Type & ALONE & vs. & HVP & vs. & KVP & vs. & HFOP \\
\hline SAME & ALONE & $>^{*}$ & $(H V P$ & $\sim$ & $K V P)$ & $>^{* *}$ & $H F O P$ \\
ONE & ALONE & $>^{* *}$ & $(H V P$ & $\sim$ & $K V P)$ & $>^{*}$ & $H F O P$ \\
OPPO & ALONE & $>^{* *}$ & $(H V P$ & $\sim$ & $K V P$ & $\sim$ & $H F O P)$ \\
\hline
\end{tabular}

comparisons have been performed to study differences in performances; they are described in Tables 8 and 9. Details of the t-tests are omitted for clarity, significant differences with a p-value less than 0.05 are signaled with a $\left(^{*}\right)$, and p-values less than 0.001 are signaled with a $\left(^{* *}\right)$. The performances are presented in Figure 3.

4.3.4 Dominance. When paired with a human, the Leader won $84.6 \%( \pm 19 \%)$ of the conflicting choices (OPPO) in the HFOP (Haptic Feedback From Object and Partner) condition. The difference between Leader and Follower dominance was statistically significant $(p<0.001, d=3.67$ ). Subjects are assigned a Leader or Follower role based on their dominance results while paired with 


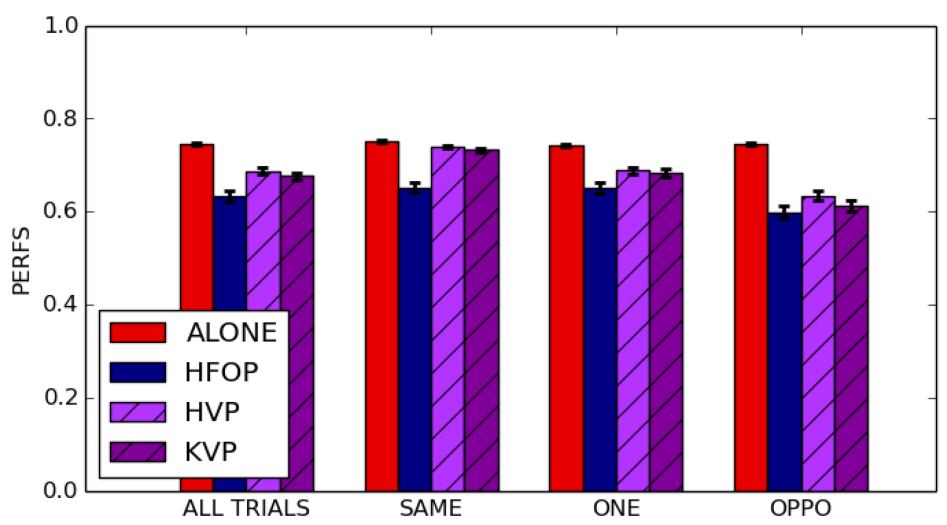

Fig. 8. Performance parameter results for the pHRI experiment. (Error bars represent standard errors of the distributions.)

a human partner, the HFOP condition, (the subject with the highest dominance is considered the leader within the dyad). Then each individual leader dominance is computed when paired with his/her own virtual partner. When the pairing is unknown (HVP condition), Leader subjects are winning $59.3 \%( \pm 28 \%)$ of the times and when the pairing with a virtual partner (KVP condition) is explicit $66 \%( \pm 32 \%)$. Compared to the HFOP dominance of $84.6 \%( \pm 19 \%)$, this is a significant drop only for HVP $(p<0.001, d=1.16)$.

The Follower subjects won $29.3 \%( \pm 25 \%)$ of the conflicting choices when unknowingly paired with the virtual partner (HVP condition), and in KVP condition they won $31.9 \%( \pm 18 \%)$. There is a statistically significant difference between the dominance of the Follower subjects in HFOP condition against both HVP and KVP conditions: HFOP vs. HVP $(p<0.001, d=-1.81)$ and HFOP vs. $\operatorname{KVP}(p<0.001, d=-1.82)$.

Furthermore, the Leader subject's dominance compared to virtual partner dominance paired with follower subjects was not statistically significant (HVP : $p=0.51, d=0.76$; KVP: $p=$ $0.09, d=1.24$ ). A "Leader" virtual partner (paired with a Follower subject) is not different than a Leader subject; their dominance cannot be separated.

4.3.5 Robot Alone. The robot controller is tailored to have similar performances to a human alone. When the virtual partner executes the task alone (ROBOT), it reaches performances similar to humans alone, but better than every other condition. (ROBOT vs. ALONE, $\mathrm{p}=0.42$; ROBOT vs. other experimental conditions, $\mathrm{p}<0.001$ ).

Average performances across decision type:

\begin{tabular}{|l|c|c|}
\hline Condition & Mean Perf & $( \pm)$ Std \\
\hline ALONE (Human alone) & 0.746 & 0.067 \\
ROBOT (Robot alone) & 0.721 & 0.110 \\
HVP (Hidden Virtual Partner) & 0.688 & 0.145 \\
KVP (Known Virtual Partner) & 0.676 & 0.157 \\
HFOP (Haptic Feedback From Object and Partner) & 0.633 & 0.141 \\
\hline
\end{tabular}

\subsection{Discussion}

In the second experiment, a virtual partner is designed based on the observation of human behavior during negotiation tasks in physical human-human interaction. The virtual partner is then tested in cooperation with human participants on the same following task previously introduced. 
The KVP and HVP conditions lead to similar performances (no significant statistical difference found between the two conditions), meaning that the a priori knowledge of the virtual nature of the partner does not influence the performances on the task (H4). The human operators tend to be more assertive when they know they operate with an artificial agent rather than a human. It is corroborated by the fact that the MAP criterion is greater in KVP than in HVP, showing a greater amount of force/movement exchanges when participants are aware that they are paired with the virtual partner. One can notice an exception in OPPO condition; this condition involves already a high level of force for obtaining an agreement. There is maybe no room for bringing more assertive motions.

The performance of the virtual partner is comparable to that of the human partners, even in conflicting situations. In more general conclusion, the performances in the HVP and KVP conditions are always equal or greater than the performances in HFOP condition (H3). Furthermore, these results do not come from higher performances of the robot guiding or leading the human, since the robot alone reaches performances similar to humans alone, as presented in Section 4.3.5.

When humans do not know they are cooperating with a virtual agent, we can observe a better performance correlated to a lower power expenditure. This result is encouraging, because it shows that it is possible to develop a virtual partner that goes beyond the human-human performances. Our results show that we might have to hide the nature of the partner to obtain a usable virtual partner; there is room for improvement.

\subsection{Study Limitations}

A limitation of this second experiment is that the force and time thresholds used for the negotiation phase with the virtual partner are fixed. While this does not seem to disturb the participants, some additional flexibility could be added in the negotiation. Those fixed thresholds, however, led to an interesting result in dominance criteria evolution: Human participants that tend to be Leader in HFOP condition stay overall Leaders in the HVP condition (although it is less pronounced). Likewise, Followers in HFOP are more dominant while paired with the virtual partner than with a Human partner.

\section{CONCLUSION}

This article presents the results of an experiment on physical Human-Human Interaction (pHHI), where human dyads cooperate on a one-dimensional comanipulative task. The results of this experiment strengthen the hypothesis that the kinesthetic channel between humans increases performances and facilitates negotiation. This extends the results of previous research work to lowimpedance tasks (zero virtual mass). Data from the pHHI experiment is used to design a virtual partner that can collaborate with humans on the same task. The virtual partner behavior is based on the observation that initiative is highly correlated to decision-making in pHHI. This virtual partner is combining a decision criteria (first crossing), a role-switching mechanism, and humanlike motions using minimum jerk strategy. This virtual partner is composed of many statistical parameters (starting time, speed, precision...) that are directly tuned from human-human observation. The virtual partner is then evaluated in a physical Human-Robot Interaction (pHRI) experiment. The results of the second experiment show that this virtual partner is able to perform the task without compromising the performances of the dyad and that a similar role distribution is observed in human-human and human-robot dyads (Followers are not becoming Leaders and vice versa). Moreover, the knowledge of the partner's nature does not seem to influence the performances even if it has some effect on the dominance of the human and the assertiveness of the human partner. The results obtained with the virtual partner are encouraging and could be used to design efficient kinesthetic controller in pHRI settings inspired from human behavior. 
The virtual partner seems to be able to successfully reproduce human behavior, both in terms of performances and decision-making. Moreover, no participant declared having detected differences between HVP and HFOP conditions when asked in post-experiment interviews. The fact that an algorithm can cooperate with humans in a task requiring physical interaction and decision-making is an encouraging step toward natural and intuitive human-robot cooperation. While the present results are obtained on a simple one degree-of-freedom task, it is a step forward in our understanding of kinesthetic channel for negotiation and its applications to physical Human-Human Interaction and physical Human-Robot Interaction. The simplicity and the controllability of this virtual partner is moreover allowing for more detailed study of the negotiation parameters.

We hypothesize that the Leader/Follower behavior of humans can be modeled by intrinsic time and force thresholds for negotiation, which vary for each person. The behavior in Hidden Virtual Partner (HVP) could explain that our tuning of the thresholds happens to be slightly lower to those of a Leader personality, but higher than those of a Follower personality. Similarly, the more dominant behavior of humans in Known Virtual Partner (KVP) condition could be explained by humans having higher thresholds when they know their partner is robotic and/or if they are more confident in the task. It thus could be possible to influence the behavior of partners in human/robot dyads by controlling the time/force thresholds available for negotiation. A study on these aspects [46] showed encouraging results consolidating this hypothesis.

The present results are of course limited to a single one-dimensional task with binary choice, and further studies are needed to test possible generalization to more complex situations. Future work will also include the generalization to others' tasks and extension to multiple degrees of freedom.

\section{REFERENCES}

[1] M. Peshkin and J. E. Colgate. 2001. Cobot architecture. IEEE Trans. Robot. Autom. 17, 4 (2001).

[2] A. De Santis, B. Siliciano, A. De Luca, and A. Bicchi. 2007. An atlas of physical human-robot interaction. Mechan. Mach. Theor. 43 (2007), 253-270.

[3] M. A. Goodrich and A. C. Schultz. 2007. Human-robot interaction: A survey. Found. Trends Hum.-Comput. Interact. 22 (2007), 70-82.

[4] N. Jarasse, V. Sanguinetti, and E. Burdet. 2013. Slaves no longer: Review on role assignment for human-robot joint motor action. Adapt. Behav. (2013).

[5] D. A. Abbink, M. Mulder, and E. R. Boer. 2012. Haptic shared control: Smoothly shifting control authority? Cogn. Tech Work 14 (2012), 19-28.

[6] D. Feth, R. Groten, A. Peer, and M. Buss. 2011. Haptic human-robot collaboration: Comparison of robot partner implementations in terms of human-likeness and task performance. Presence 20 (2011), 173-189.

[7] K. B. Reed and M. A. Peshkin. 2008. Physical collaboration of human-human and human-robot teams. IEEE Trans. Hapt. 1, 2 (2008).

[8] Y. Aydin, N. Arghavani, and C. Basdogan. 2014. A new control architecture for physical human-robot interaction based on haptic communication. In Proceedings of the ACM/IEEE International Conference on Human-robot Interaction.

[9] Y. Maeda, T. Hara, and T. Arai. 2001. Human-robot cooperative manipulation with motion estimation. In Proceedings of the International Conference on Intelligent Robots and Systems 4 (2001), 2240-2245.

[10] A. Thobbi, Y. Gu, and W. Sheng. 2011. Using human motion estimation for human-robot cooperative manipulation. In Proceedings of the IEEE/RSf International Conference on Intelligent Robots and Systems, vol. 1. 2873-2878.

[11] Paul Evrard, Elena Gribovskaya, Sylvain Calinon, Aude Billard, and Abderrahmane Kheddar. 2009. Teaching physical collaborative tasks: Object-lifting case study with a humanoid. In Proceedings of the 9th IEEE-RAS International Conference on Humanoid Robots. 399-404.

[12] P. Evrard and A. Kheddar. 2009. Homotopy switching model for dyad haptic interaction in physical collaborative tasks. In Proceedings of the 3rd foint Eurohaptics Conference and Symposium on Haptic Interfaces for Virtual Environment and Teleoperated Systems. 45-50.

[13] Alexander Mortl, Martin Lawitzky, Ayse Kucukyilmaz, Metin Sezgin, Cagatay Basdogan, and Sandra Hirche. 2012. The role of roles: Physical cooperation between humans and robots. Int. f. Robot. Res. 31, 13 (2012), 1656-1674.

[14] S. O. Oguz, A. Kucukyilmaz, T. M. Sezgin, and C. Basdogan. 2010. Haptic negotiation and role exchange for collaboration in virtual environments. In Proceedings of the IEEE Haptics Symposium. 
[15] A. Kucukyilmaz, T. M. Sezgin, and C. Basdogan. 2014. Intention recognition for dynamic role exchange in haptic collaboration. IEEE Trans. Hapt. (2014). DOI : http://dx.doi.org/10.1109/ToH.2012.21

[16] C. E. Madan, A. Kucukyilmaz, T. M. Sezgin, and C. Basdogan. 2014. Recognition of haptic interaction patterns in dyadic joint object manipulation. IEEE Trans. Hapt. 8, 1 (2014).

[17] Yanan Li, Keng Peng Tee, Wei Liang Chan, Rui Yan, Yuanwei Chua, and Dilip Kumar Limbu. 2015. Continuous role adaptation for human-robot shared control. IEEE Trans. Robot. 31 (2015), 672-681.

[18] N. Stefanov, A. Peer, and M. Buss. 2009. Role determination in human-human interaction. In Proceedings of the 3rd foint Eurohaptics Conference and Symposium on Haptic Interfaces for Virtual Environment and Teleoperator Systems. $51-56$.

[19] K. B. Reed, M. Peshkin, M. J. Hartmann, J. Patton, P. M. Vishton, and M. Grabowecky. 2006. Haptic cooperation between people, and between people and machines. In Proceedings of the IEEE/RSf International Conference on Intelligent Robots and Systems. 2109-2114.

[20] S. Glynn, R. Fekieta, and R. A. Henning. 2001. Use of force-feedback joysticks to promote teamwork in virtual teleoperation. In Proceedings of the Human Factors and Ergonomics Society 45th Annual Meeting. vol. 45. 1911-1915.

[21] G. Ganesh, A. Tagaki, T. Yoshioka, M. Kawato, and E. Burdet. 2014. Two is better than one: Physical interactions improve motor performance in humans. Nat, Sci. Rep. 4, 3824 (2014).

[22] D. De Santis, J. Znzeri, L. Masia, V. Squeri, and P. Morasso. 2014. Human-human physical interaction in the joint control of an underactuated virtual object. Conf. Proc. IEEE Eng. Med. Biol. Soc. (2014), 4407-4410.

[23] S. Gentry and E. Feron. 2005. Human-human haptic collaboration in cyclical Fitt's tasks. In Proceedings of the IEEE/RSF International Conference on Intelligent Robots and Systems. 3402-3407.

[24] J. Matsumoto and N. Inui. 2012. Two heads are better than one: Both complementary and synchronous strategies facilitate joint action. F. Neurophysiol. 109 (2012), 1307-1314.

[25] R. Ueha, H. T. T. Pham, H. Hirai, and F. Miyazaki. 2009. A simple control design for human-robot coordination based on the knowledge of dynamical role division. In Proceedings of the IEEE/RSF International Conference on Intelligent Robots and Systems. 3051-3056. DOI : http://dx.doi.org/10.1109/IROS.2009.5354109

[26] Y. Che, G. M. Haro, and A. M. Okamura. 2016. Two is not always better than one: Effects of teleoperation and haptic coupling. In Proceedings of the 6th IEEE International Conference on Biomedical Robotics and Biomechatronics (BioRob) 1290-1295. DOI : http://dx.doi.org/10.1109/BIOROB.2016.7523809

[27] C. Basdogan, C. Ho, M. A. Srinivasan, and M. Slater. 2000. An experimental study on the role of touch in shared virtual environments. ACM Trans. Comput.-hum. Interact. 7, 4 (2000), 443-460.

[28] A. Chellali, C. Dumas, and I. Milleville-Pennel. 2011. Influence of haptic communication on a shared manual task in a collaborative virtual environment. Interact. Comput. 23 (2011), 317-328.

[29] G. Avraham, I. Nisky, H. Fernandes, D. E. Acuna, K. P. Kording, and G. E. Loeb. 2012. Toward perceiving robots as humans: Three handshake models face the turing-like handshake test. IEEE Trans. Hapt. 5, 3 (2012), 196-207.

[30] J. N. Bailenson, N. Yee, S. Brave, D. Merget, and D. Koslow. 2007. Virtual interpersonal touch: Expressing and recognizing emotions through haptic devices. Hum.-comput. Interact. 22 (2007), 325-353.

[31] R. Groten, D. Feth, R. L. Klatzky, and A. Peer. 2013. The role of haptic feedback for the integration of intentions in shared task execution. IEEE Trans. Hapt. 6, 1 (2013), 94-105.

[32] J. Moll and E.-L. Sallnas. 2009. Communicative functions of haptic feedback. Hapt. Audio Interact. Des. 5763 (2009), $1-10$.

[33] A. Sawers, T. Bhattacharjee, J. L. McKay, M. E. Hackney, C. C. Kemp, and L. H. Ting. 2017. Small forces that differ with prior motor experience can communicate movement goals during human-human physical interaction. 7 . NeuroEng. Rehab. 14, 8 (2017).

[34] R. P. Van der Wel, G. Knoblitch, and N. Sebanz. 2010. Let the force be with us: Dyads exploit haptic coupling for coordination. F. Experim. Psychol.: Hum. Percept. Perform 37 (2010).

[35] C. A. C. Parker and E. A. Croft. 2011. Experimental investigation of human-robot cooperative carrying. In Proceedings of the IEEE/RSf International Conference on Intelligent Robots and Systems. 3361-3366.

[36] D. Feth, R. Groten, H. Goshy, A. Peer, D. A. Kenny, and M. Buss. 2009. Experimental analysis of dominance in haptic collaboration. In Proceedings of the 18th IEEE International Symposium on Robot and Human Interactive Communication 723-729.

[37] Atsushi Takagi, G. Ganesh, T. Yoshioka, M. Kawato, and Etienne Burdet. 2017. Physically interacting individuals estimate the partner's goal to enhance their movements. Nat. Hum. Behav. 54, 1 (2017).

[38] L. Roche and L. Saint-Bauzel. 2018. High stiffness in teleoperated comanipulation: Necessity or luxury? In Proceedings of the IEEE International Conference on Robotics and Automation. 477-483.

[39] Lucas Roche and Ludovic Saint-Bauzel. 2018. The SEMAPHORO haptic interface: A real-time low-cost open-source implementation for dyadic teleoperation. In ERTS. 
[40] Atsushi Takagi, Niek Beckers, and Etienne Burdet. 2016. Motion plan changes predictably in dyadic reaching. PLoS One 11, 12 (12 2016), 1-15. DOI : http://dx.doi.org/10.1371/journal.pone.0167314

[41] Tamar Flash and Neville Hogan. 1985. The coordination of arm movements: An experimentally confirmed mathematical model. f. Neurosci. 5, 7 (1985), 1688-1703.

[42] D. A. Lawrence. 1993. Stability and transparency in bilateral teleoperation. IEEE Trans. Robot. Autom. 9, 5 (1993), 624637.

[43] Y. Yokokohji and T. Yoshikawa. 1994. Bilateral control of master-slave manipulators for ideal kinesthetic couplingformulation and experiment. IEEE Trans. Robot. Autom. 10, 5 (1994), 605-620.

[44] M. Zhu and S. E. Salcudean. 1995. Achieving transparency for teleoperator systems under position and rate control. In Proceedings of the IEEE/RSf International Conference on Intelligent Robots and Systems, vol. 2. 7-12.

[45] E. J. Avila Mireles, J. Zenzeri, V. Squeri, P. Morasso, and D. De Santis. 2017. Skill learning and skill transfer mediated by cooperative haptic interaction. IEEE Trans. Neural Syst. Rehab. Eng. 25, 7 (July 2017), 832-843. DOI: http://dx.doi. org/10.1109/TNSRE.2017.2700839

[46] L. Roche, A. Monachan, and L. Saint-Bauzel. 2019. An experimental study of parameters influencing physical humanrobot negotiation in comanipulative tracking task. In Proceedings of the IEEE/RSfInternational Conference on Intelligent Robots and Systems (IROS). 5943-5949. DOI : http://dx.doi.org/10.1109/IROS40897.2019.8968015

[47] L. Roche and L. Saint-Bauzel. 2016. Implementation of haptic communication in comanipulative tasks: A statistical state machine model. In Proceedings of the IEEE/RSf International Conference on Intelligent Robots and Systems (IROS). 2670-2675. DOI : http://dx.doi.org/10.1109/IROS.2016.7759415

Received August 2018; revised July 2021; accepted August 2021 